\title{
A generalized class of Lyons-Zheng processes
}

\author{
FRANCESCO RUSSO ${ }^{1}$, PIERRE VALLOIS ${ }^{2}$ and JOCHEN WOLF ${ }^{1,3}$ \\ ${ }^{1}$ Institut Galilée, Mathématiques, Université Paris 13, Avenue J.B. Clément, F-93430 \\ Villetaneuse, France.E-mail: russo@math.univ-paris13.fr \\ ${ }^{2}$ Département de Mathématiques, Université de Nancy 1, B.P. 239, F-54506 Vandauvre-les- \\ Nancy, France \\ ${ }^{3}$ Fakultät für Mathematik und Informatik, Institut für Stochastik, Universität Jena, D-07740 \\ Jena, Germany.E-mail: wolf_j@minet.uni-jena.de
}

Generalizing work by Lyons and Zheng, we study Dirichlet processes admitting a decomposition into the sum of a forward and a backward local martingale plus a bounded variation process. We develop a framework of stochastic calculus for these processes and deal with existence and uniqueness for stochastic differential equations driven by such processes. In particular, Bessel processes turn out to be an interesting example of Lyons-Zheng processes.

Keywords: Bessel processes; Dirichlet processes; time reversal

\section{Introduction}

Lyons and Zhang (1994) considered a stationary symmetric good Markov process $(X(t)$, $t \geqslant 0)$ associated with a Dirichlet form and showed that, for every function $f$ belonging to the domain of the form, $f(X)$ can be represented as the sum of a forward and a backward martingale. Previously, Fukushima (1990) had shown that $f(X)$ is a Dirichlet process (see also Föllmer 1981; Bertoin 1986) for a pathwise approach), that is, it can be written as the sum of a local martingale and a zero-energy process. Thus, Lyons and Zhang (1994) gave in particular more detailed information about the zero-energy part of certain Dirichlet processes.

For us the above decomposition, which was first considered by Lyons and Zheng (1988), is the starting point for defining a class of Dirichlet processes which appear to be particularly appropriate for stochastic calculus and include time-reversible semimartingales. A Lyons-Zheng (LZ) process $X$ will be essentially the sum of a forward and a backward local martingale plus a bounded variation process. It is possible to define an LZ type of symmetric Stratonovich integral; the integrands are required to have increments which are adapted both to the future and the past of $X$. This integral does not necessarily coincide with the now classical pathwise defined symmetric Stratonovich integral which can be found, for instance, in Nualart and Pardoux (1988), Nualart (1995), Zakai (1990) and Russo and Vallois (1993). They may differ by a bracket term. We would also like to mention a first approach to this LZ type of integration by Nakao (1985) and the classical reference (Ogawa, 1984) for symmetric integration. 
This paper is organized as follows. Section 2 recalls some useful results. Sections 3 and 4 define the class of LZ processes and develop a framework for stochastic calculus. We show that this class is stable under $C^{1}$-transformations and symmetric LZ integration. We develop an Itô formula for $C^{1}$-transformations. Furthermore, we deal with existence and uniqueness for stochastic differential equations driven by an LZ process. Section 5 extends some considerations about time reversal of diffusions contained in Pardoux (1986) and Millet et al. (1989). These will be used in Section 6 to show that Bessel processes of arbitrary dimension are interesting examples of $\mathrm{LZ}$ processes.

\section{Notation and preliminaries}

In this paper $C(\mathbb{R})$ will be the Fréchet space of continuous functions on $\mathbb{R}$ endowed with the topology of uniform convergence on compacts (u.c.p.).

We fix a probability space $(\Omega, \mathscr{A}, P)$. All processes will be indexed by $[0,1]$. The Fréchet space of continuous processes equipped with the above topology is denoted by $\mathscr{C}$. We recall that a sequence of processes $\left(H_{n}\right) \in \mathscr{C}$ converges uniformly on compacts in probability (u.c.p.) to $H$ if $\sup _{t \in[0,1]}\left|\left(H_{n}-H\right)(t)\right|$ converges to zero in probability (see Protter 1990, p. 49). Note that $H$ will belong automatically to $\mathscr{C}$.

For convenience, we follow the framework of stochastic calculus introduced in Russo and Vallois (1993) and continued in Russo and Vallois $(1995 ; 1996 ; 2000)$ and Wolf $(1997 \mathrm{a}$; 1997b; 1998).

Let $X=(X(t), t \in[0,1])$ denote a continuous stochastic process and $Y=(Y(t)$, $t \in[0,1])$ a process with paths in $L^{\infty}([0,1])$. The forward, backward and symmetric integrals and the covariation process are defined by the following limits in the u.c.p. sense whenever they exist:

$$
\begin{aligned}
\int_{0}^{t} Y(s) \mathrm{d}^{-} X(s) & :=\lim _{\varepsilon \rightarrow 0+} \int_{0}^{t} Y(s) \frac{X(s+\varepsilon)-X(s)}{\varepsilon} \mathrm{d} s, \\
\int_{0}^{t} Y(s) \mathrm{d}^{+} X(s) & :=\lim _{\varepsilon \rightarrow 0+} \int_{0}^{t} Y(s) \frac{X(s)-X((s-\varepsilon) \vee 0)}{\varepsilon} \mathrm{d} s, \\
\int_{0}^{t} Y(s) \mathrm{d}^{0} X(s) & :=\lim _{\varepsilon \rightarrow 0+} \int_{0}^{t} Y(s) \frac{X(s+\varepsilon)-X((s-\varepsilon) \vee 0)}{2 \varepsilon} \mathrm{d} s, \\
{[X, Y](t) } & :=\lim _{\varepsilon \rightarrow 0+} C_{\varepsilon}(X, Y)(t),
\end{aligned}
$$

where

$$
C_{\varepsilon}(X, Y)(t):=\frac{1}{\varepsilon} \int_{0}^{t}(X(s+\varepsilon)-X(s))(Y(s+\varepsilon)-Y(s)) \mathrm{d} s .
$$

For $[X, X]$ we write $[X]$ for short. All stochastic integrals and covariation processes will, of course, be elements of $\mathscr{C}$.

For a given process $Z=(Z(t), t \in[0,1])$, we set $\hat{Z}(t):=Z(1-t), t \in[0,1]$. 
Remark 2.1. If the forward and backward integrals exist, then

(a) $\int_{0}^{t} Y(s) \mathrm{d}^{0} X(s)=\frac{1}{2} \int_{0}^{t} Y(s) \mathrm{d}^{-} X(s)+\frac{1}{2} \int_{0}^{t} Y(s) \mathrm{d}^{+} X(s)$,

(b) $[X, Y](t)=\int_{0}^{t} Y(s) \mathrm{d}^{+} X(s)-\int_{0}^{t} Y(s) \mathrm{d}^{-} X(s)$,

(c) $[X, Y](t)=[\hat{X}, \hat{Y}](1)-[\hat{X}, \hat{Y}](1-t)$.

Remark 2.2. If one of the two following integrals exists, then

$$
\int_{0}^{t} Y(s) \mathrm{d}^{+} X(s)=-\int_{1-t}^{1} \hat{Y}(s) \mathrm{d}^{-} \hat{X}(s)
$$

holds, where the integrals from $a$ to $b(a, b \in[0,1])$ are defined analogously to $(2.1)$.

Remark 2.3.

(i) If $[X, X]$ exists then it is always an increasing process and $X$ is called a finite quadratic variation process. If $[X, X] \equiv 0$, then $X$ is said to be a zero quadratic variation process (or a zero-energy process).

(ii) If $X, Y$ are continuous processes such that $[X, Y],[X, X],[Y, Y]$ exist then $[X, Y]$ has bounded (total) variation. If $f, g \in C^{1}$, then

$$
[f(X), g(Y)](t)=\int_{0}^{t} f^{\prime}(X(s)) g^{\prime}(Y(s)) \mathrm{d}[X, Y](s) .
$$

(iii) If $A$ is a zero quadratic variation process and $X$ is a finite quadratic variation process, then $[X, A] \equiv 0$.

(iv) A bounded variation process is a zero quadratic variation process.

(v) We have $[X, V] \equiv 0$ if $V$ is a bounded variation process.

In this paper all filtrations are assumed to fulfil the usual conditions. If $\mathbb{F}=\left(\mathscr{F}_{t}\right)_{t \in[0,1]}$ is a filtration, $X$ is a (continuous) $\mathbb{F}$-semimartingale and $Y$ is $\mathbb{F}$-adapted, then $\int_{0}^{t} Y \mathrm{~d}^{-} X$ is the usual Itô integral. If $Y$ is an $\mathbb{F}$-semimartingale then $\int_{0}^{t} Y \mathrm{~d}^{0} X$ is the classical FiskStratonovich integral and $[X, Y]$ the usual covariation process $\langle X, Y\rangle$.

A semimartingale $X$ such that $\hat{X}$ is again a semimartingale is said to be a time reversible semimartingale.

\section{A class of Lyons-Zheng processes}

Let $\mathbb{F}=\left(\mathscr{F}_{t}\right)_{t \in[0,1]}, \mathbb{H}=\left(\mathscr{H}_{t}\right)_{t \in[0,1]}$ be two filtrations. A process $Y=(Y(t), t \in[0,1])$ is said to be $(\mathbb{F}, \mathbb{U})$-adapted if $Y$ is $\mathbb{F}$-adapted and $\hat{Y}$ is $\mathbb{H}$-adapted. If $Y(t)-Y(0)$ is $\mathbb{F}$-adapted and $\hat{Y}(t)-\hat{Y}(0)$ is $\mathbb{M}$-adapted then we say that $Y$ is weakly $(\mathbb{E}, \mathbb{M})$-adapted.

A continuous weakly $(\mathbb{F}, \mathbb{W}$ )-adapted process $X$ is called an $(\mathbb{F}, \mathbb{U})$-Lyons-Zheng process (or simply $L Z$ process) if there are continuous processes $M^{i}=\left(M^{i}(t), t \in[0,1]\right), i=1,2$, $V=(V(t), t \in[0,1])$, such that

$$
X=\frac{1}{2} M^{1}+\frac{1}{2} M^{2}+V
$$

and the following conditions are satisfied: 


\section{Conditions 3.1.}

(i) $M^{1}$ is a local $\mathbb{F}$-martingale with $M^{1}(0)=0$.

(ii) $\hat{M}^{2}$ is a local $\mathbb{H}$-martingale with $\hat{M}^{2}(0)=M^{2}(1)=0$.

(iii) $V$ is a bounded variation process.

(iv) $M^{1}-M^{2}$ is a zero quadratic variation process.

A Lyons-Zheng process is said to be square integrable if $M^{1}, M^{2}, V \in L^{2}(\Omega \times[0,1])$.

\section{Remark 3.2.}

(i) If $X$ is an $(\mathbb{F}, \mathbb{Q})$-LZ process then $[X]=\frac{1}{2}\left(\left[M^{1}\right]+\left[M^{2}\right]\right)$ holds. In fact, Condition 3.1(iv) implies $\left[M^{1}-M^{2}\right]=0$; the bilinearity of the covariation gives $\left[M^{1}, M^{2}\right]=\frac{1}{2}\left(\left[M^{1}\right]+\left[M^{2}\right]\right)$. In particular, $X$ is a finite quadratic variation process and we have $[X]=\left[M^{1}\right]=\left[M^{2}\right]$.

(ii) If $X$ is an $(\mathbb{F}, \mathbb{H})$-LZ process then $\hat{X}$ is an $(\mathbb{H}, \mathbb{F})$-LZ process.

(iii) The decomposition (3.1) is unique. Indeed, if $\frac{1}{2} M^{1}+\frac{1}{2} M^{2}+V=0$ then (i) implies $\left[M^{1}\right]+\left[M^{2}\right]=0$. Since $M^{1}$ and $\hat{M}^{2}$ are local martingales vanishing at zero, we then have $M^{1}=M^{2} \equiv 0$.

(iv) A continuous time-reversible semimartingale is an LZ process with respect to the natural filtrations. In fact, if $X$ is a continuous $\mathbb{F}$-semimartingale and $\hat{X}$ an $\mathbb{-}$ semimartingale, then

$$
X=M^{1}+V^{1}, \quad \hat{X}=\hat{M}^{2}+\hat{V}^{2},
$$

where $M^{1}$ is an $\mathbb{F}$-local martingale with $M^{1}(0)=0$, and $\hat{M}^{2}$ is an $\mathbb{H}$-local martingale with $\hat{M}^{2}(0)=0$. Then

$$
X=\frac{1}{2} M^{1}+\frac{1}{2} M^{2}+V,
$$

where $V=\left(V^{1}+V^{2}\right) / 2$, is the LZ decomposition (3.1). Clearly, $M^{1}-M^{2}$ is a bounded variation process and thus a zero quadratic variation process.

(v) An LZ process $X$ is a Dirichlet process in the sense of Föllmer (1981) and Bertoin (1986). Indeed, we have $X=M^{1}+A$, where $A=\frac{1}{2}\left(M^{2}-M^{1}\right)+V$ is a zero quadratic variation process, being the sum of two zero quadratic variation processes.

(vi) If $X$ is a stationary symmetric Markov process associated with a Dirichlet form (see, for example, Fukushima 1990) and $u$ belongs to the domain of the form, then $u(X)$ is an LZ process (see Lyons and Zhang 1994). In this case we have $V=0$.

If $X$ is an LZ process and, moreover, $X$ and $\hat{X}$ are $\mathbb{F}$ - and $\mathbb{M}$-adapted, respectively, then $X$ is called a strong Lyons-Zheng process.

\section{Calculus with respect to $\mathrm{LZ}$ processes}

We begin this section by introducing stochastic integration with respect to an $(\mathbb{F}, \mathbb{H})$-LyonsZheng process $X$. First of all, if $Y$ is a weakly $(\mathbb{F}, \mathbb{U}$ )-adapted (continuous) process, then we define the LZ symmetric integral by 


$$
\int_{0}^{t} Y \circ \mathrm{d} X=\frac{1}{2} \int_{0}^{t} Y \mathrm{~d}^{-} M^{1}-\frac{1}{2} \int_{1-t}^{1} \hat{Y} \mathrm{~d}^{-} \hat{M}^{2}+\int_{0}^{t} Y \mathrm{~d} V
$$

We observe that

$$
\int_{0}^{t} Y \mathrm{~d}^{-} M^{1}=Y(0) M^{1}(t)+\int_{0}^{t}(Y(s)-Y(0)) \mathrm{d} M^{1}(s)
$$

so that the left-hand term is well defined. A similar consideration holds for $\int_{0}^{t} \hat{Y} \mathrm{~d}^{-} \hat{M}^{2}$. The following question immediately arises: what is the relation with the pathwise symmetric, forward and backward integrals introduced in Section 2?

We denote by $\mathscr{P}^{2}\left(\mathscr{P}^{1}\right)$ the family of weakly $(\mathbb{F}, \mathbb{H})$-adapted continuous processes $Y$ ensuring the existence of $\left[Y, M^{2}\right]\left(\left[Y, M^{1}\right]\right)$. If $Y \in \mathscr{P}^{2}$, then the forward integral of $Y$ with respect to $X$ is well defined and gives

$$
\int_{0}^{t} Y \mathrm{~d}^{-} X=\frac{1}{2} \int_{0}^{t} Y \mathrm{~d}^{-} M^{1}-\frac{1}{2} \int_{1-t}^{1} \hat{Y} \mathrm{~d}^{-} \hat{M}^{2}+\int_{0}^{t} Y \mathrm{~d} V-\frac{1}{2}\left[Y, M^{2}\right](t)
$$

If $Y \in \mathscr{P}^{1}$, then we easily see that the backward integral is well defined and we have

$$
\int_{0}^{t} Y \mathrm{~d}^{+} X=\frac{1}{2} \int_{0}^{t} Y \mathrm{~d}^{-} M^{1}-\frac{1}{2} \int_{1-t}^{1} \hat{Y} \mathrm{~d}^{-} \hat{M}^{2}+\int_{0}^{t} Y \mathrm{~d} V+\frac{1}{2}\left[Y, M^{1}\right](t) .
$$

Consequently, for $Y \in \mathscr{P}^{1} \cap \mathscr{P}^{2}$, the symmetric integral of $Y$ with respect to $X$ exists and is given by

$$
\int_{0}^{t} Y \mathrm{~d}^{0} X=\frac{1}{2} \int_{0}^{t} Y \mathrm{~d}^{-} M^{1}-\frac{1}{2} \int_{1-t}^{1} \hat{Y} \mathrm{~d}^{-} \hat{M}^{2}+\int_{0}^{t} Y \mathrm{~d} V+\frac{1}{4}\left[Y, M^{1}-M^{2}\right](t) .
$$

Comparing with (4.1) immediately yields:

Proposition 4.1. If $Y \in \mathscr{P}^{1} \cap \mathscr{P}^{2}$, then

$$
\int_{0}^{t} Y \mathrm{~d}^{0} X=\int_{0}^{t} Y \circ \mathrm{d} X+\frac{1}{4}\left[Y, M^{1}-M^{2}\right](t)
$$

If, moreover, $\left[Y, M^{1}-M^{2}\right]=0$, then

$$
\int_{0}^{t} Y \mathrm{~d}^{0} X=\int_{0}^{t} Y \circ \mathrm{d} X
$$

\section{Remark 4.2.}

(i) Since $M^{1}-M^{2}$ is a zero quadratic variation process, by Remark 2.3(iii), a sufficient condition ensuring $\left[Y, M^{1}-M^{2}\right]=0$ is the existence of $[Y]$. As an example, we mention $Y=f(X)$ for $f \in C^{1}$. In this case $\left[Y, M^{i}\right], i=1,2$, and $[Y]$ exist because of Remark 2.3(ii).

(ii) If $X$ is a time-reversible semimartingale, then, according to Remark 3.2(iv), $M^{1}-M^{2}=V^{2}-V^{1}$ is a bounded variation process. Thus, Remark 2.3(v) implies in particular that (4.3) holds for every finite quadratic variation process $Y \in \mathscr{P}^{1} \cap \mathscr{P}^{2}$. 
Take, for instance, $Y=f(X(t), t)$, where $X$ is a Brownian motion and $f$ is continuous (see Bouleau and Yor 1981; Russo and Vallois 1996; Föllmer et al. 1995).

Another situation where (4.3) holds is described in the following proposition.

Proposition 4.3. Let us suppose that $X$ is an LZ process such that $\left[f(X), M^{i}\right], i=1,2$, exist for every $f \in C^{0}(\mathbb{R})$. Then

$$
\int_{0}^{t} f(X) \mathrm{d}^{0} X=\int_{0}^{t} f(X) \circ \mathrm{d} X
$$

holds for every $f \in C^{0}(\mathbb{R})$.

Proof. The maps $f \mapsto C_{\varepsilon}\left(f(X), M^{i}\right), \varepsilon>0, i=1,2$, are continuous linear operators from $C^{0}(\mathbb{R})$ to $\mathscr{C}$. If $f \in C^{1}(\mathbb{R})$, Remark 4.2(i) tells us $\left[f(X), M^{1}-M^{2}\right]=0$. Now the BanachSteinhaus theorem for Fréchet spaces (see Dunford and Schwartz 1967, Chapter 2) yields that

$$
f \mapsto\left[f(X), M^{1}-M^{2}\right]
$$

is continuous. Thus, it must be zero since $C^{1}(\mathbb{R})$ is dense in $C^{0}(\mathbb{R})$.

LZ processes admit an Itô formula for $C^{1}$-transformations. We recall that, for timereversible semimartingales, such a formula was established in Russo and Vallois (1996) and mention that, in the special case of Brownian motion, a more general formula is stated in Föllmer et al. (1995).

Proposition 4.4. Let $X$ be an $L Z$ process. Then, for every $f \in C^{1}$, we have

$$
f(X(t))=f(X(0))+\int_{0}^{t} f^{\prime}(X) \circ \mathrm{d} X .
$$

Proof. For $f \in C^{2}(\mathbb{R}),(4.4)$ holds by Proposition 2.1 of Russo and Vallois (1995). Moreover, by definition, we have

$$
\int_{0}^{t} g(X) \circ \mathrm{d} X=\frac{1}{2} \int_{0}^{t} g(X) \mathrm{d} M^{1}-\frac{1}{2} \int_{1-t}^{1} g(\hat{X}) \mathrm{d} \hat{M}^{2}+\int_{0}^{t} g(X) \mathrm{d} V
$$

for every $g \in C^{0}(\mathbb{R})$. Thus, by localization and Doob's inequality, it is easy to verify that $g \mapsto \int_{0} g(X) \circ \mathrm{d} X$ is a continuous mapping from $C^{0}(\mathbb{R})$ to $\mathscr{C}$. Therefore (4.4) follows for every $f \in C^{1}(\mathbb{R})$ by regularizing $f$ and taking the limit.

Proposition 4.5. Let $Y$ be an $(\mathbb{F}, \mathbb{H})$-adapted process. Then

$$
Z(t)=\int_{0}^{t} Y \circ \mathrm{d} X
$$

admits a decomposition of Lyons-Zheng type (3.1). 
Proof. We can decompose $Z$ into the sum

$$
\frac{1}{2} N^{1}(t)+\frac{1}{2} N^{2}(t)+V^{0}(t)
$$

where $N^{1}(t)=\int_{0}^{t} Y \mathrm{~d}^{-} M^{1}, \quad N^{2}(t)=-\int_{t}^{1} Y \mathrm{~d}^{+} M^{2}$ and $V^{0}(t)=\int_{0}^{t} Y \mathrm{~d} V+\frac{1}{2} \int_{0}^{1} Y \mathrm{~d}^{+} M^{2}$. We observe that

$$
\hat{N}^{2}(t)=-\int_{1-t}^{1} Y \mathrm{~d}^{+} M^{2}=\int_{0}^{t} \hat{Y} \mathrm{~d}^{-} \hat{M}^{2}
$$

The decomposition in the above proposition satisfies Conditions 3.1(i)-(iii). In the following situation we also know that Condition 3.1(iv) holds.

Proposition 4.6. Let $X$ be a strong $L Z$ process and $g \in C^{1}$. Then $Z=g(X)$ is again a strong LZ process.

Proof. By the Itô formula, we have $Z(t)=g(X(0))+\int_{0}^{t} g^{\prime}(X) \circ \mathrm{d} X$ so that $Z$ admits an LZ decomposition according to Proposition 4.5.

On the other hand, $Z$ is easily seen to be a Dirichlet process with martingale part $N^{1}=$ $\int_{0}^{r} g^{\prime}(X) \mathrm{d} M^{1}$. Indeed, set $Q:=Z-N^{1}$. Then, by bilinearity, we have

$$
[Q]=[g(X)]+\left[N^{1}\right]-2\left[g(X), N^{1}\right] .
$$

By Remark 2.3(ii), we know that

$$
\begin{aligned}
{[g(X)](t) } & =\int_{0}^{t} g^{\prime}(X(s))^{2} \mathrm{~d}[X](s) \\
{\left[g(X), N^{1}\right](t) } & =\int_{0}^{t} g^{\prime}(X(s)) \mathrm{d}\left[X, N^{1}\right](s)=\int_{0}^{t} g^{\prime}(X(s))^{2} \mathrm{~d}\left[X, M^{1}\right](s), \\
{\left[N^{1}\right](t) } & =\int_{0}^{t} g^{\prime}(X(s))^{2} \mathrm{~d}\left[M^{1}\right](s) .
\end{aligned}
$$

Because of $[X]=\left[X, M^{1}\right]=\left[M^{1}\right]$, we obtain $[Q]=0$. Since $Q=\frac{1}{2}\left(N^{2}-N^{1}\right)+V^{0}$, we can conclude that $\left[N^{1}-N^{2}\right]=0$. Thus, $Z$ is an LZ process. Since it is $(\mathbb{F}, \mathbb{U})$-adapted it is also a strong $\mathrm{LZ}$ process.

We now start studying stochastic differential equations driven by an $(\mathbb{F}, \mathbb{H})$-LZ process.

Proposition 4.7. Let $\sigma:[0,1] \times \mathbb{R} \rightarrow \mathbb{R}$ be a continuous function which is Lipschitz in the second variable $x$. Let $X$ be an $(\mathbb{F}, \mathbb{U})-L Z$ process such that $\left[M^{i}\right](t)=\int_{0}^{t} Y^{i}(s) \mathrm{d} s, i=1,2$, and $V(t)=\int_{0}^{t} Y^{3}(s) \mathrm{d} s$, where $Y^{i}, i=1,2,3$, are bounded processes. Then there is at most one $(\mathbb{F}, \mathbb{M})$-adapted square-integrable process $Z$ satisfying

$$
Z(t)=y+\int_{0}^{t} \sigma(s, Z(s)) \circ \mathrm{d} X(s), \quad y \in \mathbb{R}, \quad t \in[0,1] .
$$


Proof. Let $Z_{i}, i=1,2$, be $(\mathbb{F}, \mathbb{H})$-adapted square-integrable continuous processes which are solutions to (4.5). We then have

$$
\begin{aligned}
\left(Z_{1}-Z_{2}\right)(t)= & \frac{1}{2} \int_{0}^{t} \mathrm{~d} M^{1}(s)\left(\sigma\left(s, Z_{1}(s)\right)-\sigma\left(s, Z_{2}(s)\right)\right)-\frac{1}{2} \int_{1-t}^{1} \mathrm{~d} \hat{M}^{2}(s)\left(\sigma\left(1-s, \hat{Z}_{1}(s)\right)\right. \\
& \left.-\sigma\left(1-s, \hat{Z}_{2}(s)\right)\right)+\int_{0}^{t} \mathrm{~d} V(s)\left(\sigma\left(s, Z_{1}(s)\right)-\sigma\left(s, Z_{2}(s)\right)\right) .
\end{aligned}
$$

Therefore, if $k$ is a Lipschitz constant for $\sigma$, we obtain

$$
\begin{aligned}
\mathrm{E}\left(Z_{1}-Z_{2}\right)^{2}(t) \leqslant & \frac{3 k^{2}}{4} \mathrm{E}\left(\int_{0}^{t} \mathrm{~d} s Y^{1}(s)\left|Z_{1}-Z_{2}\right|^{2}(s)\right)+\frac{3 k^{2}}{4} \mathrm{E}\left(\int_{1-t}^{1} \mathrm{~d} s Y^{2}(s)\left|\hat{Z}_{1}-\hat{Z}_{2}\right|^{2}(s)\right) \\
& +3 k^{2} \mathrm{E}\left(\int_{0}^{t} \mathrm{~d} s\left|Y^{3}\right|(s)\left|Z_{1}-Z_{2}\right|(s)\right)^{2} .
\end{aligned}
$$

So, for some constant $C$, we have

$$
\mathrm{E}\left(Z_{1}-Z_{2}\right)^{2}(t) \geqslant C \int_{0}^{t} \mathrm{~d} s \mathrm{E}\left(Z_{1}-Z_{2}\right)^{2}(s)
$$

and the conclusion follows by Gronwall's lemma.

If $\sigma$ is autonomous (i.e. depends only on $x$ ) then we can prove the existence of a solution to equation (4.5).

Proposition 4.8. Let $\sigma: \mathbb{R} \rightarrow \mathbb{R}$ be a continuous function with at most linear growth and $X a$ strong $(\mathbb{F}, \mathbb{U})-L Z$ process with $X(0)=0$. Then there exists a strong $(\mathbb{F}, \mathbb{H})-L Z$ process $Z$ solving the stochastic differential equation

$$
Z(t)=y+\int_{0}^{t} \sigma(Z(s)) \circ \mathrm{d} X(s), \quad y \in \mathbb{R}, \quad t \in[0,1] .
$$

Proof. We follow the method of Doss (1977) and Sussman (1977). Using the Peano theorem, we find locally a solution of

$$
\begin{aligned}
f^{\prime}(r) & =\sigma(f(r)), \\
f(0) & =y,
\end{aligned}
$$

for every $y \in \mathbb{R}$. This solution can be extended to the real line using the linear growth and Gronwall estimates. By the Itô formula of Proposition 4.4, $Z(t)=f(X(t))$ solves

$$
Z(t)=y+\int_{0}^{t} \sigma(Z(s)) \circ \mathrm{d} X(s) .
$$

So $Z$ is a strong LZ process in view of Proposition 4.6. 


\section{Remark 4.9.}

(i) If $\sigma$ is locally Lipschitz, another way of proving the above result on existence is to apply the Picard method using estimates of the same type as in the proof of Proposition 4.7.

(ii) Solutions to classical stochastic differential equations are semimartingales. However, the LZ processes which solve equations of type (4.5) are, in general, only Dirichlet processes and need not enjoy the semimartingale property. Indeed, in the notation of Proposition 4.8, if we choose $X$ to be a Brownian motion then, by Çinlar et al. (1980), a solution $Z$ is a semimartingale if and only if $f$ is a difference of convex functions, or equivalently, $f^{\prime}=\sigma \circ f$ has locally bounded total variation. But, if we choose a positive continuous function $\sigma$ which is not of locally bounded variation, then $\sigma \circ f$ cannot be of locally bounded variation either. So, in fact, there exist solutions to stochastic differential equations of type (4.5) that are not semimartingales.

\section{Time reversal of diffusions}

In this section we give a slight generalization of results on time reversal of diffusion processes obtained in Pardoux (1986) and Millet et al. (1989). Furthermore, we present a straightforward technique for calculating the coefficients of the time-reversed diffusion. We will exploit these results in the next section in order to study Bessel processes as LZ processes.

Let

$$
X(t)=X(0)+\int_{0}^{t} \sigma(s, X(s)) \mathrm{d} B(s)+\int_{0}^{t} b(s, X(s)) \mathrm{d} s
$$

be a diffusion with locally bounded coefficients $\sigma$ and $b$ and a Brownian motion $B$. We assume that, for every $0<t \leqslant 1$, the law of $X(t)$ has a density $p(t, x)$ with respect to Lebesgue measure.

The following theorem gives a sufficient condition ensuring that the time-reversed process is again a diffusion.

Theorem 5.1. Suppose that $\sigma$ and $b$ are jointly continuous,

$$
\sigma^{2}(s, \cdot) \in W_{\text {loc }}^{2,1}(\mathbb{R}), b(s, \cdot) \in W_{\text {loc }}^{1,1}(\mathbb{R}), x p(s, \cdot) \in W_{\text {loc }}^{2, \infty}(\mathbb{R})
$$

and

$$
\left(p \sigma^{2}\right)(s, \cdot) \in W_{\text {loc }}^{1,1}(\mathbb{R}) \text { hold for almost every } s \in[0,1]
$$

and that $\partial / \partial x\left(p \sigma^{2}\right), \partial^{2} / \partial x^{2}(x p) \in L^{1}([0, t] \times \mathbb{R}), 0<t \leqslant 1$. Then the time-reversed process $\hat{X}$ is a semimartingale and satisfies the stochastic differential equation

$$
\hat{X}(t)=X(1)+\int_{0}^{t} \sigma(1-s, \hat{X}(s)) \mathrm{d} \beta(s)+\int_{0}^{t} \tilde{b}(1-s, \hat{X}(s)) \mathrm{d} s, \quad 0 \leqslant t \leqslant 1,
$$


where $\beta$ is some Brownian motion on a possibly enlarged probability space and $\tilde{b}(s, x)=$ $-b(s, x)+\left(\partial / \partial x\left(\sigma^{2} p\right) / p\right)(s, x)$, where we set $(1 / p)(s, x):=0$ if $p(s, x)=0$.

In view of the applications below, we have chosen to base this theorem on the assumption that the law of $X(t)$ admits a density. Comparing with known results due to Pardoux (1986) and Millet et al. (1989), we remark that we do not need a Lipschitz condition on $\sigma$. In fact, the proof which uses methods of Pardoux (1986) suggests imposing regularity conditions on $\sigma^{2}$ instead of $\sigma$. This will turn out to be important for studying Bessel processes.

Lemma 5.2. Under the hypotheses of Theorem 5.1, we have the representation

$$
X(t)=X(1)+M(t)+\int_{t}^{1} \tilde{b}(s, X(s)) \mathrm{d} s,
$$

where $\hat{M}$ is a local martingale.

By changing $t$ into $1-t$ and observing

$$
[\hat{M}](t)=[\hat{X}](t)=[X](1)-[X](1-t)=\int_{0}^{t} \sigma^{2}(1-s, \hat{X}(s)) \mathrm{d} s,
$$

Theorem 5.1 follows easily from Lemma 5.2 and Theorem II.7.1' of Ikeda and Watanabe (1989).

Proof of Lemma 5.2. By a stopping argument, we may assume that $X, \sigma$ and $b$ are bounded. Let $\mathbb{H}_{s}:=\sigma\left(X_{r} ; s \leqslant r \leqslant 1\right), 0 \leqslant s \leqslant 1$. Fix $0<u<1$. We have to show that for any $t<u$,

$$
\mathrm{E}\left(X(t)-X(u)-\int_{t}^{u} \tilde{b}(s, X(s)) \mathrm{d} s \mid \mathbb{W}_{1-u}\right)=0,
$$

or equivalently, by the Markov property of $\hat{X}$,

$$
\mathrm{E}\left(X(t)-X(u)-\int_{t}^{u} \tilde{b}(s, X(s)) \mathrm{d} s \mid X(u)\right)=0 .
$$

This amounts to proving

$$
\mathrm{E}(g(X(u))(X(t)-X(u)))=\mathrm{E}\left(g(X(u)) \int_{t}^{u} \tilde{b}(s, X(s)) \mathrm{d} s\right)
$$

for every $g \in C_{c}^{\infty}$. We observe that $\mathrm{E}(g(X(u)) X(t))=\mathrm{E}(v(t, X(t)) X(t))$, where $v(t, x)=$ $\mathrm{E}(g(X(u)) \mid X(t)=x)$.

We now choose sequences $\left(\sigma_{n}\right)_{n \in \mathbb{N}}$ and $\left(b_{n}\right)_{n \in \mathbb{N}}$ of jointly continuous functions on $\mathbb{R}_{+} \times \mathbb{R}$ converging pointwise to $\sigma$ and $b$, respectively, such that, for almost every $t, \sigma_{n}(t, \cdot)$ and $b_{n}(t, \cdot)$ belong to $C^{\infty}$ and converge to $\sigma(t, \cdot)$ in $W_{\text {loc }}^{2,1}$ and $b(t, \cdot)$ in $W_{\text {loc }}^{1,1}$, respectively. By Gihman and Skorohod (1979, p. 303, Corollary 4), we then find a sequence of $C^{1,2}$ functions $v_{n}(t, x)$ solving the Cauchy problem 


$$
\begin{aligned}
\frac{\partial}{\partial t} v_{n}(t, x)+b_{n}(t, x) \frac{\partial}{\partial x} v_{n}(t, x)+\frac{1}{2} \sigma_{n}^{2}(t, x) \frac{\partial^{2}}{\partial x^{2}} v_{n}(t, x) & =0 \\
v_{n}(u, x) & =g(x), \quad x \in \mathbb{R}
\end{aligned}
$$

and satisfying $v_{n}(t, \cdot) \rightarrow v(t, \cdot)$ uniformly on compacts for every $t$. Integrating by parts and using the Itô formula, we calculate

$$
\begin{aligned}
v_{n}(t, X(t)) X(t)= & v_{n}(u, X(u)) X(u)-\int_{t}^{u} v_{n}(s, X(s)) \mathrm{d} X(s)-\int_{t}^{u} X(s) \mathrm{d} v_{n}(s, X(s)) . \\
& -\int_{t}^{u} \frac{\partial v_{n}}{\partial x}(s, X(s)) \sigma^{2}(s, X(s)) \mathrm{d} s \\
= & v_{n}(u, X(u)) X(u)-\int_{t}^{u}\left(v_{n}(s, X(s))+X(s) \frac{\partial v_{n}}{\partial x}(s, X(s))\right) \sigma(s, X(s)) \mathrm{d} B(s) \\
& -\int_{t}^{u} v_{n}(s, X(s)) b(s, X(s)) \mathrm{d} s \\
& -\int_{t}^{u} \mathrm{~d} s\left[X(s) \frac{\partial v_{n}}{\partial s}(s, X(s))+X(s) \frac{\partial v_{n}}{\partial x}(s, X(s)) b(s, X(s))\right. \\
& \left.+\frac{1}{2} X(s) \frac{\partial^{2} v_{n}}{\partial x^{2}}(s, X(s)) \sigma^{2}(s, X(s))\right]-\int_{t}^{u} \frac{\partial v_{n}}{\partial x}(s, X(s)) \sigma^{2}(s, X(s)) \mathrm{d} s .
\end{aligned}
$$

Taking expectations and using (5.3), we obtain

$$
\begin{aligned}
& \mathrm{E}\left(v_{n}(t, X(t)) X(t)\right)=\mathrm{E}(g(X(u)) X(u))-\mathrm{E}\left(\int_{t}^{u} v_{n}(s, X(s)) b(s, X(s)) \mathrm{d} s\right) \\
& \quad-\int_{t}^{u} \mathrm{~d} s \int_{\mathbb{R}} \mathrm{d} x p(s, x) x\left[\frac{\partial v_{n}}{\partial x}(s, x)\left(b(s, x)-b_{n}(s, x)\right)+\frac{1}{2} \frac{\partial^{2} v_{n}}{\partial x^{2}}(s, x)\left(\sigma^{2}(s, x)-\sigma_{n}^{2}(s, x)\right)\right] \\
& \quad-\int_{t}^{u} \mathrm{~d} s \int_{\mathbb{R}} \frac{\partial v_{n}}{\partial x}(s, x) \sigma^{2}(s, x) p(s, x) \mathrm{d} x \\
& \mathrm{E}(g(X(u)) X(u))-\mathrm{E}\left(\int_{t}^{u} v_{n}(s, X(s)) b(s, X(s)) \mathrm{d} s\right) \\
& \quad+\int_{t}^{u} \mathrm{~d} s \int_{\mathbb{R}} \mathrm{d} x\left[v_{n}(s, x) \frac{\partial}{\partial x}\left(x p(s, x)\left(b(s, x)-b_{n}(s, x)\right)\right)\right. \\
& \left.\quad-\frac{1}{2} v_{n}(s, x) \frac{\partial^{2}}{\partial x^{2}}\left(x p(s, x)\left(\sigma^{2}(s, x)-\sigma_{n}^{2}(s, x)\right)\right)\right] \\
& \quad+\int_{t}^{u} \mathrm{~d} s \int_{\mathbb{R}} \mathrm{d} x \boldsymbol{v}_{n}(s, x) \frac{\partial}{\partial x}\left(p(s, x) \sigma^{2}(s, x)\right) .
\end{aligned}
$$


In the limit as $n \rightarrow \infty$, by hypothesis, we obtain

$$
\begin{aligned}
\mathrm{E}(g(X(u)) X(t))= & \mathrm{E}(g(X(u)) X(u))-\mathrm{E}\left(g(X(u)) \int_{t}^{u} b(s, X(s)) \mathrm{d} s\right) \\
& +\mathrm{E}\left(g(X(u)) \int_{t}^{u}\left(\frac{\partial}{\partial x}\left(p \sigma^{2}\right) / p\right)(s, X(s)) \mathrm{d} s\right) .
\end{aligned}
$$

We now present a technique for calculating the drift coefficient of the time reversal of a diffusion. This works in the following setting: let

$$
X(t)=X(0)+\int_{0}^{t} \sigma(s, X(s)) \mathrm{d} B(s)+\int_{0}^{t} b(s, X(s)) \mathrm{d} s
$$

be a diffusion with locally bounded coefficients $\sigma, b$ such that the law of $X(t)$ admits a density $p(t, x)$ for every $t>0$. We suppose that the time-reversed process $\hat{X}$ is again a diffusion. It then takes the form

$$
\hat{X}(t)=X(1)+\int_{0}^{t} \sigma(1-s, \hat{X}(s)) \mathrm{d} \beta(s)+\int_{0}^{t} \tilde{b}(1-s, \hat{X}(s)) \mathrm{d} s .
$$

By a localization argument, we may suppose $b$ and $\sigma$ to be bounded. The technique for calculating $b$ basically consists of evaluating the backward integral $\int_{0}^{t} f(X) \mathrm{d}^{+} X$ for $f \in C^{1}$ with compact support in two different ways. First, we calculate

$$
\begin{aligned}
\int_{0}^{t} f(X(s)) \mathrm{d}^{+} X(s)= & \int_{0}^{t} f(X(s)) \mathrm{d}^{-} X(s)+[f(X), X](t) \\
= & \int_{0}^{t} f(X(s)) \sigma(s, X(s)) \mathrm{d} B(s) \\
& +\int_{0}^{t} f(X(s)) b(s, X(s)) \mathrm{d} s+\int_{0}^{t} f^{\prime}(X(s)) \sigma^{2}(s, X(s)) \mathrm{d} s .
\end{aligned}
$$

Then we compute

$$
\begin{aligned}
\int_{0}^{t} f(X(s)) \mathrm{d}^{+} X(s) & =-\int_{1-t}^{1} f(\hat{X}(s)) \mathrm{d}^{-} \hat{X}(s) \\
& =-\int_{1-t}^{1} f(\hat{X}(s)) \sigma(1-s, \hat{X}(s)) \mathrm{d} \beta(s)-\int_{1-t}^{1} f(\hat{X}(s)) \tilde{b}(1-s, \hat{X}(s)) \mathrm{d} s .
\end{aligned}
$$

Identifying the expectations of (5.4) and (5.5) yields

$$
\int_{0}^{t} \mathrm{~d} s \int_{\mathbb{R}} \mathrm{d} x p(s, x)\left[f(x) b(s, x)+f^{\prime}(x) \sigma^{2}(s, x)+f(x) \tilde{b}(s, x)\right]=0 .
$$

Integrating by parts, we obtain 


$$
\int_{0}^{t} \mathrm{~d} s \int_{\mathbb{R}} \mathrm{d} x f(x)\left[p(s, x)(b+\tilde{b})(s, x)-\frac{\partial}{\partial x}\left(p \sigma^{2}\right)(s, x)\right]=0,
$$

provided that $p \sigma^{2}$ is absolutely continuous and its density $\partial / \partial x\left(p \sigma^{2}\right)$ is locally integrable on $[0, \infty) \times \mathbb{R}$. By a density argument, we conclude

$$
\tilde{b}(s, x)=\left(\frac{\partial}{\partial x}\left(p \sigma^{2}\right) / p\right)(s, x)-b(s, x),
$$

where we set $(1 / p)(s, x):=0$ if $p(s, x)=0$.

Finally, we consider the example of square Bessel processes. Let us recall that a square Bessel process of dimension $\delta \geqslant 0$ starting at 0 is characterized as the unique solution to the stochastic differential equation

$$
X(t)=2 \int_{0}^{t}(X(s))^{1 / 2} \mathrm{~d} B(s)+\delta t
$$

where $B$ is a Brownian motion. Since the law of $X(t)$ has the density

$$
p(t, x)=(2 t)^{-\delta / 2} \Gamma(\delta / 2)^{-1} x^{\delta / 2-1} \exp (-x / 2 t)
$$

(see Revuz and Yor 1994, Section XI.1), we obtain by the above arguments that the timereversed process $\hat{X}$ is a solution to the stochastic differential equation

$$
\hat{X}(t)=X(1)+2 \int_{0}^{t}(\hat{X}(s))^{1 / 2} \mathrm{~d} \beta(s)-8 \int_{0}^{t} \frac{\hat{X}(s)}{1-s} \mathrm{~d} s+\delta t .
$$

\section{Example: Bessel processes of arbitrary dimension}

For $\delta \geqslant 1$, the Bessel process is a semimartingale and can be characterized as a solution to some stochastic differential equation. For $\delta<1$ it does not enjoy the semimartingale property but remains a Dirichlet process (see, for example, Yor 1997). We prove that, for arbitrary dimension $\delta>0$, a Bessel process is in fact a Lyons-Zheng process.

We first aim to understand the impact of the dimension $\delta$ on the structure of the Bessel process. We fix $\Delta>0$. Using equation (5.7) and applying the Itô formula, we obtain

$$
\sqrt{X(t)+\Delta}=\sqrt{\Delta}+\int_{0}^{t}\left(\frac{X(s)}{X(s)+\Delta}\right)^{1 / 2} \mathrm{~d} B(s)+\frac{\delta}{2} \int_{0}^{t} \frac{\mathrm{d} s}{(X(s)+\Delta)^{1 / 2}}-\frac{1}{2} \int_{0}^{t} \frac{X(s)}{(X(s)+\Delta)^{3 / 2}} \mathrm{~d} s .
$$

If $\delta>1$, letting $\Delta \rightarrow 0$, we arrive at the stochastic differential equation describing the Bessel process $Y:=\sqrt{X}$ :

$$
Y(t)=B(t)+\frac{\delta-1}{2} \int_{0}^{t}(Y(s))^{-1} \mathrm{~d} s .
$$

The convergence is basically justified by $\mathrm{E}\left(\int_{0}^{t}(X(s))^{-1 / 2} \mathrm{~d} s\right)<\infty$. 
If $\delta \leqslant 1$, however, we have $\mathrm{E}\left(\int_{0}^{t}(X(s))^{-1 / 2} \mathrm{~d} s\right)=\infty$. Consequently, (6.2) no longer holds because $\mathrm{E}(Y(t))<\infty$ and $\mathrm{E}(|B(t)|)<\infty$. We cannot even apply the Itô formula in the general setting of Russo and Vallois (1996) to describe $\sqrt{X}$ if $\delta \leqslant 1$ since then $\left[X^{-1 / 2}, X\right]$ does not exist. To see this, we show that the defining approximations $C_{\varepsilon}\left(X^{-1 / 2}, X\right)$ do not exist as integrable random variables. We have

$$
\begin{aligned}
C_{\varepsilon}\left(X^{-1 / 2}, X\right)(t)= & \frac{1}{\varepsilon} \int_{0}^{t} X(s+\varepsilon)^{-1 / 2} X(s+\varepsilon) \mathrm{d} s \\
& +\frac{1}{\varepsilon} \int_{0}^{t} X(s)^{-1 / 2} X(s) \mathrm{d} s-\frac{1}{\varepsilon} \int_{0}^{t} X(s+\varepsilon)^{-1 / 2} X(s) \mathrm{d} s-\frac{1}{\varepsilon} \int_{0}^{t} X(s)^{-1 / 2} X(s+\varepsilon) \mathrm{d} s .
\end{aligned}
$$

Since the law of $X(s)$ is absolutely continuous with respect to Lebesgue measure we have $\int_{0}^{t} \mathbf{1}_{\{X(s)=0\}} \mathrm{d} s=0$ almost surely. Therefore, the first two terms are finite. Hence, it is sufficient to show

$$
\mathrm{E}\left(X(s)^{-1 / 2} X(s+\varepsilon)\right)=+\infty
$$

Using the Markov property of the square Bessel process, we calculate

$$
\begin{aligned}
\mathrm{E}\left(X(s)^{-1 / 2} X(s+\varepsilon)\right) & =\mathrm{E}\left(X(s)^{-1 / 2} X(s+\varepsilon) \mid \mathscr{F}_{s}\right)=\mathrm{E}\left(X(s)^{-1 / 2} \mathrm{E}(X(s+\varepsilon) \mid X(s))\right) \\
& =\mathrm{E}\left(X(s)^{-1 / 2}(X(s)+\delta \varepsilon)\right)=\mathrm{E}\left(X(s)^{1 / 2}\right)+\delta \varepsilon \mathrm{E}\left(X(s)^{-1 / 2}\right) .
\end{aligned}
$$

The first term is finite, the second infinite. This establishes (6.3).

The basic difficulty that, for $\delta \leqslant 1,(6.1)$ contains a difference of two terms converging to $+\infty$ can be eliminated by using Stratonovich calculus:

$$
\begin{aligned}
\sqrt{X(t)+\Delta} & =\sqrt{\Delta}+\frac{1}{2} \int_{0}^{t}(X(s)+\Delta)^{-1 / 2} \mathrm{~d}^{0} X(s) \\
& =\sqrt{\Delta}+\frac{1}{4} \int_{0}^{t}(X(s)+\Delta)^{-1 / 2} \mathrm{~d}^{-} X(s)+\frac{1}{4} \int_{0}^{t}(X(s)+\Delta)^{-1 / 2} \mathrm{~d}^{+} X(s) .
\end{aligned}
$$

Using Remark 2.2 and equations (5.7) and (5.8), we obtain 


$$
\begin{aligned}
\sqrt{X(t)+\Delta}= & \sqrt{\Delta}+\frac{1}{4} \int_{0}^{t}(X(s)+\Delta)^{-1 / 2} \mathrm{~d} X(s)-\frac{1}{4} \int_{1-t}^{1}(\hat{X}(s)+\Delta)^{-1 / 2} \mathrm{~d}^{-} \hat{X}(s) \\
= & \sqrt{\Delta}+\frac{1}{2} \int_{0}^{t}\left(\frac{X(s)}{X(s)+\Delta}\right)^{1 / 2} \mathrm{~d} B(s)+\frac{\delta}{4} \int_{0}^{t} \frac{\mathrm{d} s}{(X(s)+\Delta)^{1 / 2}} \\
& -\frac{1}{2} \int_{1-t}^{1}\left(\frac{\hat{X}(s)}{\hat{X}(s)+\Delta}\right)^{1 / 2} \mathrm{~d} \beta(s)+2 \int_{1-t}^{1} \frac{\hat{X}(s)}{(\hat{X}(s)+\Delta)^{1 / 2}(1-s)} \mathrm{d} s \\
& -\frac{\delta}{4} \int_{1-t}^{1} \frac{\mathrm{d} s}{(\hat{X}(s)+\Delta)^{1 / 2}} \\
= & \sqrt{\Delta}+\frac{1}{2} \int_{0}^{t}\left(\frac{X(s)}{X(s)+\Delta}\right)^{1 / 2} \mathrm{~d} B(s) \\
& -\frac{1}{2} \int_{1-t}^{1}\left(\frac{\hat{X}(s)}{\hat{X}(s)+\Delta}\right)^{1 / 2} \mathrm{~d} \beta(s)+2 \int_{0}^{t} \frac{X(s)}{s(X(s)+\Delta)^{1 / 2}} \mathrm{~d} s,
\end{aligned}
$$

where $B$ and $\beta$ are the driving Brownian motions in the stochastic differential equations (5.7) and (5.8). In the limit as $\Delta \rightarrow 0$, we see that $Y$ is the sum of a forward and backward martingale plus a bounded variation process. In fact it is truly its Lyons-Zheng decomposition.

Proposition 6.1. A Bessel process $Y$ of arbitrary dimension is a Lyons-Zheng process with LZ decomposition

$$
Y(t)=\sqrt{X(t)}=\frac{1}{2} B(t)-\frac{1}{2}(\beta(1)-\beta(1-t))+2 \int_{0}^{t} \frac{Y(s)}{s} \mathrm{~d} s .
$$

Proof. Since (6.4) has already been established it only remains to verify that $[B+(\beta(1)-$ $\beta(1-\cdot))]=0$. We restrict ourselves to $\delta<1$, the semimartingale case being easier. By Yor (1997, Section 10.1), we know that the canonical decomposition of $Y$ as a Dirichlet process is given by

$$
Y_{t}=B_{t}+\frac{\delta-1}{2} \mathrm{p} \cdot \mathrm{v} \cdot \int_{0}^{t} \frac{1}{Y_{s}} \mathrm{~d} s .
$$

In the part of zero quadratic variation, the principal value

$$
\text { p.v. } \int_{0}^{t} \frac{1}{Y_{s}} \mathrm{~d} s=\int_{\mathbb{R}} x^{-1} \mathbf{1}_{(0, \infty)}(x)\left(L^{s(Y)}(t, s(x))-L^{s(Y)}(t, 0)\right) m(\mathrm{~d} x)
$$

is defined using scale function $s$ and speed measure $m$ of $Y$ and the local time $L^{s(Y)}$ of $s(Y)$ (see also Engelbert and Wolf 1998, Section 4.1 - to make sure that we may deal with the same $B$ in (5.7), (6.4) and (6.5). Combining (6.4) and (6.5)), we conclude that 


$$
B_{t}+\beta(1)-\beta(1-t)=(1-\delta) \text { p.v. } \int_{0}^{t} \frac{1}{Y_{s}} \mathrm{~d} s+4 \int_{0}^{t} \frac{Y_{s}}{s} \mathrm{~d} s
$$

has zero quadratic variation.

Remark 6.2. Relation (6.6) sheds some light on the striking fact that the dimension $\delta$ does not appear in the LZ decomposition (6.4). Actually, we deduce from (6.6) that

$$
\beta_{t}=B_{1-t}-B_{1}+(1-\delta) \text { p.v. } \int_{1-t}^{1} \frac{1}{Y_{S}} \mathrm{~d} s+4 \int_{1-t}^{1} \frac{Y_{s}}{S} \mathrm{~d} s
$$

depends on $\delta$. Thus, the influence of the dimension $\delta$ on the LZ decomposition (6.4) is implicitly included in the Brownian motion $\beta$.

\section{Acknowledgements}

The research of the third author has been supported by TMR grant ERBFMBICT971937 of the European Union.

\section{References}

Bertoin, J. (1986) Les processus de Dirichlet en tant qu'espace de Banach. Stochastics, 18, 155-168. Bouleau, N. and Yor, M. (1981) Sur la variation quadratique des temps locaux de certaines semimartingales. C. R. Acad. Sci. Paris Sér. I, 292, 491-494.

Çinlar, E., Jacod, J., Protter, P. and Sharpe, M.J. (1980) Semimartingales and Markov processes. Z. Wahrscheinlichkeitstheorie Verw. Geb., 54, 161-219.

Doss, H. (1977) Liens entre équations différentielles stochastiques et ordinaires. Ann. Inst. H. Poincaré (n. Sér.) Sect. B, 13, 99-125.

Dunford, N. and Schwartz, J.T. (1967) Linear Operators, Part I: General Theory. New York: Wiley.

Engelbert, H.J. and Wolf, J. (1998) Strong Markov local Dirichlet processes and stochastic differential equations. Teor. Veroyatnost. i Primenen., 43, 331-348.

Föllmer, H. (1981) Calcul d'Itô sans probabilités. In J. Azéma and M. Yor (eds), Séminaire de Probabilités XV, Lecture Notes in Math. 850, pp. 143-150. Berlin: Springer-Verlag.

Föllmer, H., Protter, P. and Shiryayev, A.N. (1995) Quadratic covariation and an extension of Itô's formula. Bernoulli, 1, 149-169.

Fukushima, M. (1990) Dirichlet Forms and Markov Processes. Amsterdam: North-Holland.

Gihman, I.I. and Skorohod, A.V. (1979) The Theory of Stochastic Processes III. Berlin: SpringerVerlag.

Ikeda, N. and Watanabe, S. (1989) Stochastic Differential Equations and Diffusion Processes. Amsterdam: North-Holland.

Lyons, T.J. and Zhang, T.S. (1994) Decomposition of Dirichlet processes and its applications. Ann. Probab., 22, 494-524.

Lyons, T.J. and Zheng, W. (1988) A crossing estimate for the canonical process on a Dirichlet space and a tightness result. Colloque Paul Lévy sur les processus stochastiques, Astérisque, 157-158, 249-271. 
Millet, A., Nualart, D. and Sanz, M. (1989) Integration by parts and time reversal of diffusion processes. Ann. Probab., 17, 208-238.

Nakao, S. (1985) Stochastic calculus for continuous additive functionals of zero energy. $Z$. Wahrscheinlichkeitstheorie Verw. Geb., 68, 557-578.

Nualart, D. (1995) The Malliavin Calculus and Related Topics. New York: Springer-Verlag.

Nualart, D. and Pardoux, E. (1988) Stochastic calculus with anticipating integrands. Probab. Theory Related Fields, 78, 535-581.

Ogawa, S. (1984) Une remarque sur l'approximation de l'intégrale stochastique du type non-causal par une suite d'intégrales de Stieltjes. Tôhoku Math. J., 36, 41-48.

Pardoux, E. (1986) Grossissement d'une filtration et retournement d'une diffusion. In J. Azéma and M. Yor (eds), Séminaire de Probabilités XX, Lecture Notes in Math. 1204. Berlin: SpringerVerlag.

Protter, P. (1990) Stochastic Integration and Differential Equations. Berlin: Springer-Verlag.

Revuz, D. and Yor, M. (1994) Continuous Martingales and Brownian Motion. Berlin: Springer-Verlag.

Russo, F. and Vallois, P. (1993) Forward, backward and symmetric stochastic integration. Probab. Theory Related Fields, 97, 403-421.

Russo, F. and Vallois, P. (1995) The generalized covariation process and Itô formula. Stochastic Process. Appl., 59, 81-104.

Russo, F. and Vallois, P. (1996) Itô formula for $\mathrm{C}^{1}$-functions of semimartingales. Probab. Theory Related Fields, 104, 27-41.

Russo, F. and Vallois, P. (2000) Stochastic calculus with respect to a finite quadratic variation process. Stochastics Stochastics Rep., 70, 1-40.

Sussman, H.J. (1977) An interpretation of stochastic differential equations as ordinary differential equations which depend on a sample point. Bull. Amer. Math. Soc., 83, 296-298.

Wolf, J. (1997a) Transformations of semimartingales and local Dirichlet processes. Stochastics Stochastics Rep., 62, 65-101.

Wolf, J. (1997b) An Itô formula for continuous local Dirichlet processes. Stochastics Stochastics Rep., 62, 103-115.

Wolf, J. (1998) A representation theorem for continuous additive functionals of zero quadratic variation. Probab. Math. Statist., 18, 367-379.

Yor, M. (1997) Some Aspects of Brownian Motion, Part II: Some New Martingale Problems. Basel: Birkhäuser-Verlag.

Zakai, M. (1990) Stochastic integration, trace and skeleton of Wiener functionals. Stochastics, 33, 93-108.

Received January 1999 and revised May 2000 